\title{
Point-of-Care Tests for Severe Hemorrhage - A Manual for Diagnosis and Treatment
}

\author{
Marco Ranucci, Paolo Simioni (editors). Springer, 2016. ISBN 978-3-319-24795-3
}

\author{
Carlos Corredor, MD
}

Received: 12 April 2016/Accepted: 19 April 2016/Published online: 27 April 2016

(C) Canadian Anesthesiologists' Society 2016

Critically ill patients can present clinicians with challenging, often life-threatening, derangements in hemostasis and coagulation. The authors of Point-of-Care Tests for Severe Hemorrhage are members of the Italian Network to Address Coagulopathy Treatment (INTACT) group, which comprises a multidisciplinary group of Italian clinicians with interest and expertise in this field. This book is the first edition and reflects the efforts of several authors to compile relevant and practical information on the use of point-of-care coagulation tests (POCTs) in the setting of severe hemorrhage.

The book features 14 chapters, with the initial six chapters setting the theoretical background for the pathophysiology of coagulation, historical perspective of POCTs, general aspects of viscoelastic and platelet function tests, and blood products. Subsequent chapters provide practical information on the use of POCTs applied to clinical scenarios frequently associated with severe bleeding, such as cardiovascular surgery, postpartum hemorrhage, trauma, liver surgery, and in the intensive care unit. At 211 pages, the book is reasonably sized given the length and depth of topics covered.

Chapters have wide variability in layout and writing style, which likely reflects the large number of contributory authors. They are not uniformly structured, with only some containing clearly defined introduction and conclusion sections. There is also considerable variation in chapter length. The variability, however, does not affect the flow of the book. The text is easy to read, and the authors make frequent use of tables, which are particularly

C. Corredor, MD ( $\square)$

Toronto General Hospital, Toronto, ON, Canada

e-mail: carloscorredor@ doctors.org.uk helpful in summarizing the data presented. Color illustrations are also used effectively throughout the book, helping the reader understand the concepts and clinical scenarios presented in the text. The reader can also refer to current and comprehensive reference lists present in each chapter.

The initial chapter provides a complete, yet succinct, overview of the pathophysiology of coagulation. Understanding the complex interactions between the various cellular and coagulation proteins that comprise the hemostatic system is aided by clear illustrations and diagrams. Clinical correlation with congenital and acquired hemostatic disorders and their effects, as reflected in traditional clotting laboratory tests, is a particularly helpful feature.

The technical aspects, similarities, differences, and limitations of multiple viscoelastic coagulation and platelet function POCTs are explained in detail in the following three chapters. The authors of these chapters successfully describe the inner working of these POCTs in a way that is understandable to the nonlaboratory-based clinician. A very short chapter, only two pages long, deals with the history of POCTs being introduced into clinical practice. It is well written, and its historical aspects are relevant. I did wonder, however, about the merit of presenting it as a separate chapter.

The use of blood products, derivatives, and prohemostatic drugs in the context of severe hemorrhage is covered in an excellent chapter. The authors explain how the various blood products are obtained and list the indications for their use. Use of anti-fibrinolytic agents, fibrinogen, and factor concentrates are also reviewed using an evidence-based approach. I found it particularly useful that the use of POCTs for monitoring the effect of each of 
these blood products or pharmacological agent is explained in detail.

The second half of the book describes the management of severe bleeding and use of POCTs in specific patient populations. The section on management of severe bleeding in trauma patients summarizes well the current state of our understanding of the pathophysiology of trauma-induced coagulopathy. The authors recommend methods for monitoring and managing severe bleeding related to trauma based on a thorough review of the available literature and international guidelines. The chapter gives a detailed overview of the Early Coagulation Support Protocol adopted by the Italian Trauma Network.

Cardiac surgery is responsible for the use of a large portion of blood and blood products in many countries. Therefore, a chapter dedicated to severe bleeding in cardiovascular patients is of particular importance. Although bleeding mechanisms during both adult and pediatric cardiac surgery and the use of viscoelastic and platelet function tests were addressed, the authors could have explained and illustrated in greater detail how, in practice, POCTs are integrated into practice using the multimodal protocol algorithms and transfusion strategies that are now recommended by international guidelines.

The chapter on postpartum hemorrhage reviews key aspects of the pathophysiology and the role that POCTs can play in the management of a condition responsible for significant maternal morbidity and mortality. The authors of the chapter on liver disease and transplantation cover the concept of 'rebalanced hemostasis' in patients with liver disease and provide a rationale for the use of POCTs, instead of conventional coagulation tests, as the POCTs are more dynamic and reflective of the rebalanced state. Other clinical scenarios of coagulopathy in the critically ill patient, such as sepsis and burns, are discussed in a separate chapter.

There is also a chapter that explores the role of POCTs in monitoring the effects of traditional antithrombotic drugs such as heparin and vitamin $\mathrm{K}$ antagonists, as well as the increasingly used novel oral anticoagulants.

Chapters dedicated to logistical and quality control issues surrounding the incorporation of POCTs into clinical practice, and clinical cases illustrating the use of POCTs, close this manual.

In summary, the first edition of this manual successfully achieved the objectives of the authors in compiling current, comprehensive, and relevant information on POCTs and their application in various severe hemorrhage scenarios. This textbook will appeal to a broad readership of trainees and clinicians of different disciplines involved in the management of this challenging group of patients.

Conflicts of interest None declared.

Editorial responsibility This submission was handled by Dr. Hilary P. Grocott, Editor-in-Chief, Canadian Journal of Anesthesia. 